\section{Stethoscope modification}

To the Editor:

I would like to present a modification of one's personal stethoscope, consisting of the insertion of a Luer connector between the ear set and the stethoscope head (Figure). This arrangement was devised to provide an adequate and comfortable ear set, always immediately available, for use with oesophageal stethoscopes. Also, when access is possible, the stethoscope head can be left taped to the chest and monitored as needed. This improves oesophageal and precordial chest auscultation, inasmuch as the ear set and stethoscope head are of a higher quality than that of the equipment usually available in the operating room. Another benefit of this arrangement is that one may vary the length of the tubing at will to suit specific monitoring needs or personal preference.

Lastly, in emergency situations, the male end of the Luer connector is truly instantly available to set up a device for percutaneous transtracheal ventilation similar to that described previously.' The connector can be fitted to the fresh gas hose from the anaesthesia machine, or to a length of standard oxygen tubing, and attached to the hub of a 14 gauge intravenous catheter inserted into the trachea. Ventilation is carried out by intermittently activating the flush system on the anaesthesia machine. ${ }^{1}$

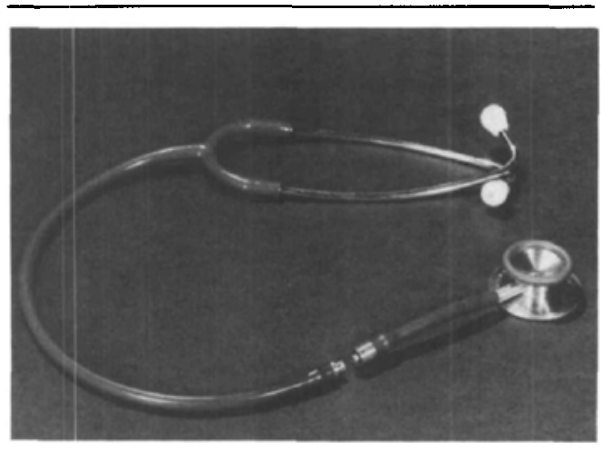

FIGURE 1 Modified stethoscope. Note that the male end of the luer connector is on the side of the stethoscope head.
The basic modification allows a greater versatility of this common piece of equipment in everyday practice. The Luer connector itself may prove invaluable in an emergency situation.

Jean-François Hardy MD

Resident in Anaesthesia

Université de Montréal

5000 est, rue Bélanger

Montréal, Québec, HIT 1C8

\section{REFERENCE}

1 Scuderi PE, McLeskey CH, Comer PB. Emergency percutaneous transtracheal ventilation during anesthesia using readily available equipment. Anesth Analg 1982; 61: 867-70.

\section{Uptake of nitrous oxide by man}

To the Editor:

I wish to comment on the recent article by Virtue $e t$ $a l .{ }^{1}$ concerning the uptake of nitrous oxide in man. The rate of uptake of nitrous oxide in man was first described in 1954 by Severinghaus who estimated uptake to be above $1000 \mathrm{ml}$ in the first minute with a rapid reduction to a level of $150 \mathrm{ml} \cdot \mathrm{min}^{-1}$ within the next few minutes and approximated by the power function $1000 \times \mathrm{t}^{-\frac{1}{2}} .^{2}$ This rate of uptake was measured at the mouthpiece and not at the alveolar membrane, however, so that the effect of wash-in into the functional residual capacity of the lung was overlooked. The functional residual capacity of the lung in a given patient is a relatively fixed noncollapsible space and the wash-in of any anaesthetic gas, such as nitrous oxide, takes place according to a simple exponential function. This wash-in should not be considered as body uptake. However, true body uptake of inhalation anaesthetics takes place across the alveolar membrane and closely follows Fick's principle of diffusion which, in this case, indicates that the uptake depends on the inspired concentration-mixed venous concentration difference, on the existence of a membrane factor, and on cardiac output. $^{5}$ 